\title{
The prognostic value of regadenoson stress: Has the case been made?
}

\author{
Rami Doukky, MD, MSc, FACC, FASNC ${ }^{\mathrm{a}, \mathrm{b}}$ \\ a Division of Cardiology, John H. Stroger, Jr. Hospital of Cook County, Chicago, IL \\ b Division of Cardiology, Rush University Medical Center, Chicago, IL
}

Received May 11, 2015; accepted May 11, 2015

doi: $10.1007 /$ s12350-015-0179-3

\section{See related article, pp. 600-607}

Since its approval by the Food and Drug Administration in April of 2008, regadenoson has enjoyed a wide implementation by nuclear cardiology laboratories. Presently, it is the preferred vasodilator stress agent in the United States, being used in 8 out of 10 pharmacologic stress SPECT myocardial perfusion imaging (MPI) studies. ${ }^{1}$ The initial approval of regadenoson was based on two phase 3 ADVANCE-MPI trials, in which regadenoson was demonstrated to produce perfusion defects similar to those induced by standard 6 minute adenosine infusion. ${ }^{2,3}$ Although diagnostic noninferiority of regadenoson was effectively demonstrated, prognostic noninferiority was beyond the scope of the ADVANCE-MPI trials. Nonetheless, given the similarity in perfusion images, the prognostic value of regadenoson stress was presumed to parallel that of adenosine.

In nuclear cardiology, a field that prides itself on vast wealth of outcomes data, extending the prognostic utility of adenosine to regadenoson is just not good enough. In recent years, several studies have investigated the prognostic value of regadenoson stress MPI. Iqbal et al compared the 2-year outcome of patients with normal regadenoson MPI $(n=1,000)$ to those with normal adenosine MPI $(n=1,000)$ for the composite endpoint of cardiac death, myocardial infarction, and coronary revascularization. ${ }^{4}$ The annual event rate was

Reprint requests: Rami Doukky, MD, MSc, FACC, FASNC Division of Cardiology, John H. Stroger, Jr. Hospital of Cook County, 1901 W. Harrison St., Suite \# 3620, Chicago, IL, 60612; rami_doukky @ rush.edu

J Nucl Cardiol 2015;22:608-10.

$1071-3581 / \$ 34.00$

Copyright (C) 2015 American Society of Nuclear Cardiology.
$1.1 \%$ in the regadenoson group and $1.7 \%$ in the adenosine group $(P=0.090)$ and the annual cardiac death rate was $0.9 \%$ and $1.15 \%(P=0.404)$, respectively. In propensity score matched analysis of 505 patients from each group, the respective annual event rates in the regadenoson and adenosine groups were $0.7 \%$ and $1.3 \%$ $(P=0.257)$ for the primary outcome, and $0.5 \%$ and $0.7 \%(P=0.763)$ for cardiac death. Thus, the study effectively demonstrated that, as with normal adenosine MPI, a normal regadenoson MPI predicts very low event rates. ${ }^{4}$ More recently, Hage et al evaluated the prognostic value of regadenoson stress in 1,400 subjects (700 consecutive normal MPI and 700 consecutive abnormal MPI). ${ }^{5}$ Based on the quantitative perfusion defect size (percentage of left ventricular myocardium), the cohort was divided into four groups: Group 1, normal $(<5 \%)$; Groups 2, small defect $(<10 \%)$; Group 3, moderate sized defect (10\%-20\%); Group 4, large defect (>20\%). During a mean follow-up of 4 years, the composite endpoint of cardiac death, myocardial infarction, and late coronary revascularization ( $>90$ days after MPI) occurred in 10, 27, 31, and 43\% ( $P<0.001)$; while early revascularization ( $<90$ days) occurred in $0.4,9$, 17 , and $17 \%$ in Groups $1-4$, respectively $(P<0.001)$. The authors demonstrated a step-wise increase in event rates commensurate with the size of regadenoson-induced defect. The study showed that, as with other stress agents, regadenoson MPI provides powerful prognostic information which can guide clinical decision-making. ${ }^{5}$

In this issue of the journal, Farzaneh-Far et al compared the prognostic value of regadenoson and adenosine MPI in 3,698 consecutive patients $(1,737$ adenosine and 1,961 regadenoson) followed for 1 year. $^{6}$ The cohort included patients with normal and abnormal MPI, unlike the study by Iqbal et al which analyzed patients with normal MPI. The investigators used inverse probability weighted Cox proportional hazards regression modeling to balance the baseline characteristics associated with each vasodilator agent. Irrespective of the vasodilator choice, the Summed 
Stress Score (SSS) remained a significant predictor of the composite endpoint of cardiovascular death or myocardial infarction (hazard ration [HR], 1.36 per $5 \%$ increment; $95 \%$ confidence interval [CI] 1.28-1.46; $P<0.0001)$ and cardiovascular death (HR, 1.38 per $5 \%$ increment; CI 1.28-1.49; $P<0.0001)$. Similarly, the Summed Difference Score (SDS) was a significant predictor of the composite of cardiovascular death and myocardial infarction (HR, 1.61 per 5\% increment; CI 1.38-1.88; $P<0.001)$ and cardiovascular death (HR 1.46 per $5 \%$ increment; CI $1.22-1.75$; $P<0.001)$. Importantly, there was no significant interaction between SSS and stress agent or between SDS and stress agent as determinants of any of the study endpoints. In other words, SSS and SDS derived from adenosine or regadenoson MPI rendered similarly significant prognostic value. This is an important finding; it affirms comparable prognostic significance of perfusion images produced by regadenoson and adenosine. In this study, differences in baseline characteristics between the adenosine and regadenoson cohorts were addressed using inverse probability weighted Cox regression models. This technique balances the disparate burden in baseline characteristics by weighting each patients according to the inverse of the probability of receiving or not receiving a stress agent. Weighting ensures that, for any combination of baseline characteristics (leading to any propensity score value), the sum of contributions of patient-disease burden from either study group is equal. This technique can be advantageous over traditional multivariate Cox regression modeling when there are too many covariates to adjust for or when the number of events are too few, thus risking model over-fitting. Unlike the propensity score matching technique, inverse probability weighting maintains the full sample in the analysis. The authors appropriately implemented this technique to address the differences in the baseline characteristics between the study groups without losing power to propensity score matching or risking over-fitting their Cox models. Inverse probability weighting is underutilized in nuclear cardiology outcomes research, and it should be considered when appropriate.

In the present study and the one by Iqbal and colleagues, it is remarkable that the regadenoson cohorts were substantially lower risk than the adenosine cohorts. This was evident in lower prevalence of coronary disease and coronary risk factors with a trend toward lower event rates observed in the regadenoson groups. ${ }^{4,6}$ In the present study, this imbalance in risk also manifested in lower SSS burden and higher mean left ventricular ejection fraction in the regadenoson group. ${ }^{6}$ It is interesting to observe a downtrend in disease burden within the span of 2-3 years separating adenosine from regadenoson eras. There are at least two plausible explanations for this phenomenon. First, it may be related to the downtrend in the burden of myocardial ischemia and coronary disease burden which has been reported in recent literature. ${ }^{7,8}$ Second, this observation may be due to MPI testing of lower risk population over the years, coinciding with the switch from adenosine to regadenoson. Testing a lower risk population may be directly related to the switch to regadenoson. The convenience of regadenoson administration may have tempted clinicians to use vasodilator stress more liberally in lower risk patients who may be candidates for exercise stress. It is well established that patients undergoing exercise stress MPI are generally healthier with fewer comorbidities and are at lower risk for adverse events than those undergoing pharmacologic stress. ${ }^{9}$ The use of regadenoson stress in patients who are able to exercise may be a contributing factor to the observed lower coronary disease burden among patients undergoing regadenoson stress. In this regard, we should be reminded that exercise stress modality is preferred for the vast majority of patients and we should not trade the clinical and prognostic data derived from exercise stress for the convenience of regadenoson use.

Not only do perfusion images derived from regadenoson yield similar prognostic information to adenosine, but also the physiologic response to regadenoson provides comparable prognostic data. As is the case with adenosine stress, a blunted heart rate response to regadenoson vasodilator stress has been shown to be an independent prognostic indicator in patients undergoing myocardial perfusion imaging. ${ }^{10,11}$ In a study of 1,156 patients followed for a mean of 22 months, Hage et al demonstrated that heart rate response to regadenoson in the lowest quartile $(<17 \%$ increase from baseline) was independently associated with a five-fold increase in mortality compared to the highest quartile ( $>43 \%$ increase from baseline), after adjusting for age, gender, diabetes mellitus, renal disease, and MPI findings. More recently, our group demonstrated that, in patients selected for coronary angiography, regadenoson-induced electrocardiographic ST-segment depression is associated with increased prevalence of severe coronary artery disease and higher rates of major adverse cardiac events, independent of clinical covariates and MPI findings. In a deviation from the literature, the diagnostic value of transient ischemic dilation with regadenoson seems to be questionable, as shown by Golzar et al. ${ }^{12,13}$ Despite the breadth of regadenoson published data, there remain small gaps in the literature. Although a limited number of small studies demonstrated the safety and tolerability of regadenoson in patients with end-stage renal disease, ${ }^{14-16}$ the use of regadenoson in this population remains offlabel with no published outcomes data. 
After a few years of clinical regadenoson use, a formidable body of literature has been published, establishing regadenoson as the new standard in vasodilator stress. The present investigation cemented the prognostic utility of regadenoson stress, demonstrating that perfusion abnormalities produced by regadenoson carry similar prognostic information as with adenosine. Based on the present study and others, the case for the prognostic value of regadenoson stress is well made.

\section{Disclosures}

The author receives research funding from Astellas Pharma and served on the Advisory Board for Astellas Pharma.

\section{References}

1. Astellas Pharma US. Lexiscan ${ }^{\circledR}$. http://www.lexiscan.com. Accessed May 8, 2015.

2. Iskandrian AE, Bateman TM, Belardinelli L, Blackburn B, Cerqueira MD, Hendel RC, et al. Adenosine versus regadenoson comparative evaluation in myocardial perfusion imaging: Results of the ADVANCE phase 3 multicenter international trial. J Nucl Cardiol 2007; 14:645-58.

3. Mahmarian JJ, Peterson LE, Xu J, Cerqueira MD, Iskandrian AE, Bateman TM, et al. Regadenoson provides perfusion results comparable to adenosine in heterogeneous patient populations: A quantitative analysis from the ADVANCE MPI trials. J Nucl Cardiol 2015;22:248-61.

4. Iqbal FM, Hage FG, Ahmed A, Dean PJ, Raslan S, Heo J, et al. Comparison of the prognostic value of normal regadenoson with normal adenosine myocardial perfusion imaging with propensity score matching. JACC Cardiovasc Imag 2012;5:1014-21.

5. Hage FG, Ghimire G, Lester D, McKay J, Bleich S, El-Hajj S et al. The prognostic value of regadenoson myocardial perfusion imaging. J Nucl Cardiol 2015. doi:10.1007/s12350-014-0050-y.

6. Farzaneh-Far A, Shaw LK, Dunning A, Oldand JD, O'Connor $\mathrm{CM}$, Borges-Neto S. Comparison of the prognostic value of regadenoson and adenosine myocardial perfusion imaging. J Nucl Cardiol 2015. doi:10.1007/s12350-015-0155-y.

7. Rozanski A, Gransar H, Hayes SW, Min J, Friedman JD, Thomson LE, et al. Temporal trends in the frequency of inducible myocardial ischemia during cardiac stress testing: 1991 to 2009. J Am Coll Cardiol 2013;61:1054-65.

8. Duvall WL, Rai M, Ahlberg AW, O'Sullivan DM, Henzlova MJ. A multi-center assessment of the temporal trends in myocardial perfusion imaging. J Nucl Cardiol 2015. doi:10.1007/s12350014-0051-x.

9. Poulin MF, Alexander S, Doukky R. Prognostic implications of stress modality on mortality risk and cause of death in patients undergoing office-based SPECT myocardial perfusion imaging. J Nucl Cardiol 2015. doi:10.1007/s12350-014-0064-5.

10. Hage FG, Dean P, Iqbal F, Heo J, Iskandrian AE. A blunted heart rate response to regadenoson is an independent prognostic indicator in patients undergoing myocardial perfusion imaging. J Nucl Cardiol 2011;18:1086-94.

11. Iqbal FM, Al Jaroudi W, Sanam K, Sweeney A, Heo J, Iskandrian $\mathrm{AE}$, et al. Reclassification of cardiovascular risk in patients with normal myocardial perfusion imaging using heart rate response to vasodilator stress. Am J Cardiol 2013;111:190-5.

12. Golzar Y, Olusanya A, Pe N, Dua SG, Golzar J, Gidea C et al. The significance of automatically measured transient ischemic dilation in identifying severe and extensive coronary artery disease in regadenoson, single-isotope technetium-99 m myocardial perfusion SPECT. J Nucl Cardiol 2015. doi:10.1007/s12350-015-0087-6.

13. Bourque JM. Contemporary relevance of TID: Based on the company it keeps. J Nucl Cardiol 2015. doi: 10.1007/s12350-015-0122-7.

14. Aljaroudi W, Hermann D, Hage F, Heo J, Iskandrian AE. Safety of regadenoson in patients with end-stage renal disease. Am J Cardiol 2010;105:133-5.

15. Doukky R, Rangel MO, Wassouf M, Dick R, Alqaid A, Morales DR. The safety and tolerability of regadenoson in patients with end-stage renal disease: The first prospective evaluation. J Nucl Cardiol 2013;20:205-13.

16. Rangel MO, Morales Demori R, Voll ST, Wassouf M, Dick R, Doukky R. Severe chronic kidney disease as a predictor of benefit from aminophylline administration in patients undergoing regadenoson stress myocardial perfusion imaging: A substudy of the ASSUAGE and ASSUAGE-CKD trials. J Nucl Cardiol 2014. doi: 10.1007/s12350-014-0036-9. 